\title{
Escolhas metodológicas em investigação científica: aplicação da abordagem de Saunders no estudo da influência da cultura na competitividade de clusters
}

Fátima Regina Teixeira르, Luiz Roberto $\mathrm{Mayr}^{2}$, António Viera Paisana ${ }^{3}$, Filipa Dionísio Vieira ${ }^{3}$

fatima@ifsc.edu.br, lrmayr@inmetro.gov.br, apaisana@dps.uminho.pt, filipadv@dps.uminho.pt

\begin{abstract}
${ }^{1}$ Programa Doutoral em Engenharia Industrial e Sistemas, Universidade do Minho, 4804 - 533, Guimarães, Portugal

${ }^{2}$ Núcleo de Gestão Ambiental, Instituto Nacional de Metrologia, Qualidade e Tecnologia, Av. N. S. das Graças 50, prédio 24, 25250-020, Duque de Caxias, Brasil
\end{abstract}

3 Departamento de Produção e Sistemas, Universidade do Minho, 4804 - 533, Guimarães, Portugal

DOI: 10.17013/risti.n.85-98

\begin{abstract}
Resumo: Este artigo tem por objetivo apresentar, com base em Saunders et al., as escolhas metodológicas que são adotadas na investigação científica acerca da influência da cultura local sobre a cultura organizacional e a competitividade, no estudo do cluster da malacocultura da região da Grande Florianópolis, situado no sul do Brasil. Saunders considera que são as sucessivas escolhas do investigador em relação às filosofias, à abordagem, às estratégias, aos métodos de coleta $\mathrm{e}$ análise de dados, ao horizonte de tempo e às técnicas e procedimentos de coleta $\mathrm{e}$ análise de dados, que caracterizam a investigação científica. O trabalho busca demonstrar que evidenciar as escolhas metodológicas é uma forma de reflexão que possibilita aumentar significativamente a consistência do modelo da investigação.
\end{abstract}

Palavras-chave: escolhas metodológicas; modelo de investigação; cluster.

Methodological choices in scientific research: use of the Saunders approach on the study of the culture influence on clusters competitivity

\begin{abstract}
This article aims to present, based on Saunders et al., the methodological choices that are adopted in a scientific research about the influence of local culture on organizational culture and competitiveness, in a study on the malacoculture cluster of Florianópolis, in southern Brazil. Saunders believes that the successive choices of the researcher in relation to philosophies, approach, strategies, methods of collection and analysis of data, time horizon and techniques and procedures for collecting and analyzing data are what characterize the scientific research. The paper aims to demonstrate that showing the methodological choices is a form of reflection which enables to significantly increase the consistency of the research model.
\end{abstract}


Keywords: qualitative methods; case study; cluster.

\section{Introdução}

No Departamento de Produção e Sistemas, da Escola de Engenharia da Universidade do Minho está em curso um trabalho de investigação sobre o cluster da malacoculutra da região da Grande Florianópolis, no Sul do Brasil. Este cluster, voltado ao cultivo de moluscos bivalves marinhos, compartilha condições geográficas bastante favoráveis pela qualidade da água de baía, e conta com apoio institucional de organizações como a Universidade Federal de Santa Catarina (UFSC) e a Empresa de Pesquisas Agropecuárias e Extensão Rural de Santa Catarina (EPAGRI), entre outras. O cluster apresenta como resultado a geração de emprego e renda na região, a preservação da cultura local e o cuidado com o meio ambiente.

Para Porter (1998), cluster é a concentração geográfica de empresas interconectadas, fornecedores especializados, provedores de serviços, empresas em setores correlatos e instituições associadas em campos específicos, que tanto competem como cooperam entre si. Os clusters podem ser um meio para o alcance de competitividade pelo compartilhamento de conhecimentos entre os envolvidos e pelo estímulo à inovação. A dualidade entre competição e cooperação é uma necessidade de sobrevivência das empresas diante de questões comuns no mercado, e requer uma cultura organizacional favorável. No entanto, as especificidades da cultura local podem favorecer ou dificultar a formação de um ambiente propício a esta simultaneidade de competição com cooperação entre empresas, afetando a sua competitividade. A competitividade nesse caso pode ser compreendida como a capacidade de uma organização ou nação de ser bem sucedida em relação à concorrência (Mariotto, 1991). Investigar como os clusters se organizam é uma forma de contribuir para a consolidação deste instrumento de competitividade e desenvolvimento regional.

Embora contando com apoio institucional, com condições ambientais favoráveis e com ambiente econômico propício, o cluster da malacocultura da região da Grande Florianópolis apresenta problemas. Minuzzi (2011) observou que alguns dos fatores que dificultam o pleno desenvolvimento do cluster estão relacionados ao comportamento dos indivíduos, à sua maneira de ser e agir, que é condicionada por sua cultura. A autora afirma que entre os principais pontos fracos do cluster na percepção dos membros da governança endógena estão, entre outros, a rivalidade entre os produtores, a diversidade de interesses entre os membros, a falta de entendimento do conceito de cluster, a ausência de espírito de união, cooperação e associativismo entre os produtores, e a dificuldade de comunicação entre os agentes.

A falta de cooperação, de união e de espírito associativo identificados por Minuzzi (2011) no cluster da malacocultura da região da Grande Florianópolis pode ser entendida como uma manifestação de individualismo, talvez um traço da herança cultural açoriana dos colonizadores dessa região, quem sabe uma característica comum desenvolvida pelos indivíduos da localidade ao longo de suas vidas na atividade prévia da pesca artesanal extrativa, que é muitas vezes realizada de forma individual, isolada. 
Dentre os problemas, ou entraves ao desenvolvimento do cluster da malacocultura da Grande Florianópolis apresentados por Minuzzi (2011) percebe-se que a maior parte está relacionada ao comportamento dos indivíduos, ou seja, à sua maneira de ser e agir, que, por sua vez, está condicionada pela sua cultura. Assim, por exemplo, a falta de cooperação, de união e de espírito associativo entre os produtores poderia ser entendida como uma manifestação de individualismo, quem sabe um traço da herança açoriana que colonizou a região, quem sabe uma característica comum aos produtores, desenvolvida ao longo de suas vidas como pescadores, já que muitos são originários da pesca artesanal, atividade extrativa e, muitas vezes, de caráter individual.

No âmbito dos estudos sobre organizações, inúmeros trabalhos abordam a relação entre cultura local e cultura organizacional. Neles, os autores reconhecem a influência da cultura local sobre a organizacional, assim como os seus reflexos no desempenho das organizações. Alguns, por exemplo, buscam compreender a relação entre cultura organizacional e cultura nacional (Hofstede, 1980, 1990; Lenartowicz, 2001; Mcsweeney, 2009), outros estão mais interessados no estudo da liderança, numa complexa relação entre cultura nacional, cultura organizacional e eficácia de práticas de liderança (Dickson et al., 2000; House et al., 2010).

A cultura organizacional tem sido citada por vários autores como um dos aspectos da maior relevância no que se refere à competitividade das empresas. Diversos estudos reconhecem a importância da cultura no desempenho organizacional, como os de Barney (1986), de Baird et al. (2007), e de Martins e Terblanche (2003). Para Fairbanks e Lindsay (2000), é a cultura que formata o que os indivíduos pensam sobre riscos, recompensas, oportunidades e, consequentemente, sobre progresso. Feldman (2010), por sua vez, mostra que os fatores culturais influenciam a gestão das empresas e, consequentemente, afetam o seu desempenho, destacando a necessidade de se ter consciência de que não adianta lutar contra estes fatores, mas aprender a tirar proveito deles. No entanto, percebe-se que existem aspectos relativos às especificidades características da região, que são ligados à cultura local e, ao mesmo tempo, passíveis de afetar a competitividade de clusters, que não são abordados nestes estudos, mas que caberia investigá-los.

Analisar os efeitos da cultura local na cultura organizacional, no desempenho das empresas e na competitividade de um cluster envolve lidar com aspectos subjetivos, não quantificáveis, em uma investigação que combina conhecimentos sobre clusters, competitividade e cultura organizacional, baseados em Porter (1998, 2008), Hofstede (1980) e Schein (1984, 1985). O desafio inicial de uma investigação dessa natureza é estabelecer o método e os procedimentos adequados para a coleta e análise de dados. Para Saunders et al. (2012), o investigador deve ser capaz de refletir sobre as suas escolhas e justificá-las em relação às alternativas que poderia ter adotado; são as suas escolhas que irão caracterizar a investigação.

Este artigo tem por objetivo apresentar, com base na abordagem de Saunders et al. (2012), as escolhas metodológicas que são adotadas na investigação acerca dos efeitos da cultura local sobre a cultura organizacional e competitividade de clusters. Organizado em cinco secções este trabalho apresenta na sua primeira secção a contextualização do problema; na seção 2, a fundamentação teórica em torno dos aspectos metodológicos; na seção 3, a proposição da abordagem metodológica a ser utilizada; na seção 4, um esboço do modelo de coleta e análise de dados a ser adotado; 
e, finalmente, na sua última secção, apresenta uma avaliação prévia da adequação do modelo aos objetivos da investigação. Espera-se com este artigo contribuir para a discussão sobre a aplicação de métodos qualitativos em investigações na área da Engenharia.

\section{Fundamentação teórica}

Saunders et al. (2012) afirmam que em investigação científica a metodologia constituise no conjunto de procedimentos intelectuais e técnicos utilizados pelo investigador para alcançar os seus objetivos. A metodologia adotada para uma investigação deixa transparecer a visão de mundo do investigador por meio dos pressupostos filosóficos e paradigmáticos que ele elege e que estarão na base do novo conhecimento construído. Os autores consideram que mais importante do que declarar a filosofia ou o paradigma que norteiam a sua investigação é o investigador ser capaz de refletir sobre as suas escolhas. Assim, apresentam um modelo para estruturar a investigação a partir de alternativas em relação às filosofias, à abordagem, às estratégias, aos métodos de coleta e análise de dados, ao horizonte de tempo e às técnicas e procedimentos de coleta $\mathrm{e}$ análise de dados. Segundo eles, são as sucessivas escolhas do investigador em relação a cada um destes aspectos que irão caracterizar a investigação.

\section{Filosofia}

Segundo Saunders et al. (2012), as quatro correntes filosóficas em investigação científica, o positivismo, o realismo, o interpretativismo e o pragmatismo distinguemse em suas perspectivas ontológicas, epistemológicas e axiológicas.

Ontologicamente, isto é, do ponto de vista do investigador quanto à natureza da realidade ou do ser, tanto o positivismo como o realismo consideram que a realidade é objetiva e que é independente dos atores sociais. Já o interpretativismo considera que a realidade é subjetiva, uma construção social que pode mudar. A corrente pragmática, por sua vez, considera que o ponto de vista do investigador deve ser escolhido de forma a melhor responder à pergunta de investigação (Saunders et al., 2012).

Epistemologicamente, isto é, quanto ao tipo de conhecimento que é aceitável, tanto o positivismo como o realismo consideram que apenas os fenômenos observáveis podem prover dados confiáveis para a investigação, sendo que o primeiro enfoca na casualidade e em generalizações, e o segundo na explicação dentro de um determinado contexto. Já o interpretativismo considera os significados subjetivos e o fenômeno social com foco nos detalhes da situação, a realidade por trás desses detalhes e os significados subjetivos que motivam os atores sociais. A corrente pragmática considera que ambos, fenômenos observáveis e significados subjetivos, podem prover conhecimentos aceitáveis, dependendo da questão de investigação (Saunders et al., 2012).

Axiologicamente, isto é, quanto ao papel dos valores em investigação científica, no positivismo a investigação é livre dos valores do investigador que, independentemente dos dados, mantém uma postura objetiva. No realismo, a investigação é carregada dos valores do investigador, suas visões, culturas e experiências, que terão impacto na investigação. No interpretativismo, a investigação é de valor vinculado, e o investigador 
é parte do que está sendo investigado. Finalmente, na corrente pragmática, os valores desempenham um grande papel na interpretação dos resultados, sendo que neste caso o investigador pode adotar pontos de vista tanto objetivos quanto subjetivos (Saunders et al., 2012).

\section{Abordagem}

Segundo Saunders et al. (2012), existem duas formas possíveis de abordagens em investigação científica: a indutiva e a dedutiva. Na dedutiva, o investigador formula uma teoria e hipóteses e estabelece uma estratégia de investigação para testar e validar essas hipóteses, levando à possibilidade de generalização; é a abordagem dominante no campo das ciências naturais. $\mathrm{Na}$ indutiva, o investigador coleta dados e formula a teoria como resultante da análise de dados, isto é, o estabelecimento da regra ou a formulação da teoria decorre do fenômeno ou efeito observado; é a abordagem dominante no campo das ciências sociais. Para os autores, se o interesse do investigador é maior no sentido de compreender por que alguma coisa está acontecendo do que em descrever o que acontece, deve usar a abordagem indutiva de investigação. Consideram que a abordagem indutiva tende a se preocupar com o contexto em que os eventos ocorrem. Por isso, o estudo de pequenas amostras do objeto pode ser mais apropriado do que de grandes amostras, como ocorre na abordagem dedutiva.

\section{Estratégia}

Saunders et al. (2012) consideram que para definir a estratégia a ser adotada em investigação científica é necessário que antes se defina a finalidade da investigação. Segundo os autores, a investigação pode ter a finalidade exploratória, descritiva e explanatória. A investigação exploratória é um meio para descobrir o que está acontecendo, fazer perguntas e avaliar a situação sob uma nova perspectiva. A investigação descritiva serve para traçar o perfil de pessoas, eventos ou situações. Já a explanatória ou explicativa é a que busca estabelecer relações causais entre variáveis.

Diversas são as estratégias que podem ser adotadas em processos de investigação científica: o experimento, o inquérito, o estudo de caso, a investigação-ação, a teoria fundamentada, a etnografia e a investigação em arquivos (Saunders et al., 2012).

O experimento, como estratégia, serve a estudos que envolvam ligações causais; costuma ser usado em investigações explanatórias e exploratórias. O inquérito é uma estratégia popular, pois permite grande coleta de dados de modo econômico e costuma ser usado em investigações exploratórias e descritivas. O estudo de caso permite uma rica compreensão do contexto de uma investigação e dos processos encenados, e costuma ser usado em investigações explanatórias e exploratórias. A investigação-ação combina coleta de dados e facilitação para a mudança. A teoria fundamentada é particularmente útil em investigações de previsão e explicação de comportamento para a criação de uma teoria. A etnografia, por sua vez, busca descrever e explicar o mundo social que os sujeitos da investigação vivenciam, da maneira como eles próprios o descrevem e explicam. Finalmente, a investigação em arquivos é a estratégia que usa documentos e registros administrativos como principal fonte de dados e permite que 
questões com foco sobre o passado e mudanças ao longo do tempo possam ser respondidas (Saunders et al., 2012).

\section{Coleta e análise de dados}

De acordo com Saunders et al. (2012), há duas escolhas possíveis quanto aos métodos para a coleta e análise de dados em investigação científica: o mono-método e o método múltiplo. O mono-método, como o próprio termo indica, é a opção por uma única técnica para a coleta e análise de dados. Já o método múltiplo é a opção pelo uso de mais de uma técnica para a coleta e análise de dados. Nesse caso, a escolha pode ser por multi-método, que consiste na opção pelo uso combinado de diferentes técnicas qualitativas ou no uso combinado de diferentes técnicas quantitativas de coleta $\mathrm{e}$ análise de dados ou a escolha pode ser pelo uso do método misto, que consiste na combinação de diferentes técnicas quantitativas e qualitativas de coleta e análise de dados.

\section{Horizonte de tempo}

Segundo Saunders et al. (2012), o horizonte de tempo em investigação científica pode ser tanto longitudinal quanto transversal, e depende da questão de investigação. $\mathrm{O}$ estudo longitudinal é o que permite estudar mudança e desenvolvimento ao longo de um período de tempo. Já o estudo transversal é o que permite o estudo de um determinado fenômeno, em um determinado momento.

\section{Técnicas e Procedimentos}

De acordo com Saunders et al. (2012), as técnicas de amostragem em investigação científica dividem-se em dois tipos: as probabilísticas, ou representativas e as não probabilísticas, ou de julgamento. Nas técnicas de amostragem do tipo probabilísticas a amostra é escolhida estatisticamente e de forma aleatória; nelas é possível especificar a probabilidade de que qualquer das alternativas existentes seja incluída na amostra. As técnicas de amostragem probabilísticas resumem-se em aleatória simples, aleatória sistemática, aleatória estratificada, grupo e multi estágio, cada qual possuindo uma fórmula matemática específica para a seleção. Já na amostragem do tipo não probabilística há uma variedade de técnicas que podem ser utilizadas na seleção da amostra, que é feita com base em julgamento subjetivo e, portanto, não probabilístico. As técnicas de amostragem não probabilísticas resumem-se em: cota, intencional caso extremo, intencional heterogênea, intencional homogênea, intencional caso crítico, intencional caso típico, intencional teórica, voluntária bola de neve, voluntária auto seleção e acidental por conveniência.

Em relação aos procedimentos a serem utilizados para a coleta e análise de dados primários, Saunders et al. (2012) citam a observação, a entrevista semiestruturada e o questionário como instrumentos de coleta. Para a análise dos dados os autores apresentam duas formas possíveis de análise: a qualitativa e a quantitativa.

No que concerne especificamente aos procedimentos de coleta, a observação pode ser participante ou estruturada e envolve praticamente a observação sistemática, a gravação, a análise, a descrição e a interpretação do comportamento das pessoas. A 
observação participante é qualitativa e busca descobrir os significados que as pessoas atribuem às suas ações. A observação estruturada, por sua vez, é quantitativa e está relacionada à frequência com que algo ocorre. A entrevista semiestruturada, é utilizada em investigações qualitativas em que o investigador terá uma lista de questões e temas a serem cobertas, que podem variar de entrevistado para entrevistado. Finalmente, o questionário, é o que corresponde para os autores e no contexto de seu livro, a todas as técnicas de coleta de dados em que pessoas são solicitadas a responder a um mesmo conjunto de questões, em uma mesma sequência predeterminada, incluindo a entrevista estruturada (Saunders et al., 2012).

No que concerne especificamente à análise de dados, a quantitativa envolve a interpretação de dados numéricos padronizados, com base em diagramas e cálculos estatísticos, que permitem examinar a relação entre variáveis. A questão crítica neste tipo de análise é o tratamento dos dados para permitir o seu correto processamento e interpretação de resultados. Já a análise qualitativa é baseada em dados que são expressos através de palavras; informações não padronizadas que demandam classificação em categorias. Nesse tipo de análise o desafio é a definição dos conceitos que permitem a análise dos dados. A análise qualitativa pode ser dedutiva ou indutiva. A dedutiva é realizada com base em teoria pré-existente. Já na indutiva, a discussão teórica emerge do processo de coleta e análise de dados (Saunders et al., 2012).

\section{Proposição}

Com base no modelo de Saunders et al. (2012), a presente investigação insere-se na corrente filosófica do interpretativismo, que leva em consideração os significados subjetivos que motivam a ação individual e os fenômenos sociais. O conhecimento da realidade se dará pela interação entre a investigadora e os atores sociais que integram o objeto de análise, durante o processo de investigação.

A abordagem usada para a investigação é a indutiva, prevalecendo a preocupação com o contexto dos acontecimentos. Consiste em estudo explanatório, em que se busca estabelecer relações causais entre variáveis. A explanação acerca do fenômeno estudado decorrerá, portanto, dos dados coletados e da sua análise.

A estratégia adotada é o estudo de caso, tomando-se como objeto de análise o cluster da malacocultura da região da Grande Florianópolis. Yin (2009) considera que a aplicação mais importante do estudo de caso como estratégia investigativa é para explicar ligações causais presumidas em intervenções da vida real que são muito complexas para estratégias como o inquérito ou o experimento. Os estudos de caso, assim como os experimentos, são generalizáveis para proposições teóricas e não para universos e populações. Eles não representam uma amostra. A decisão de utilizar o estudo de caso como estratégia de investigação deve ocorrer quando o investigador quer entender um fenômeno da vida real em profundidade, mas tal compreensão engloba condições contextuais altamente pertinentes ao fenômeno estudado.

A coleta de dados será realizada por meio do método múltiplo, do tipo multimétodo qualitativo, em que se combinam diferentes técnicas qualitativas de coleta, tendo-se em vista que a pesquisa busca compreender os diversos aspectos subjetivos que afetam o 
objeto do estudo, de modo a tornar possível, pela análise qualitativa, explanar acerca da situação existente e inferir a seu respeito. A opção pelo uso de mais de uma técnica qualitativa para a coleta de dados permite que a investigadora possa, por esse tipo de análise, explanar acerca da realidade encontrada e inferir a seu respeito, já que o interesse está em compreender os aspectos subjetivos que afetam o objeto de estudo.

Quanto ao horizonte de tempo, a investigação em curso caracteriza-se como um estudo transversal, já que o fenômeno é estudado durante um tempo determinado.

No que concerne à técnica de amostragem, será adotada a técnica não probabilística de amostragem, que é a indicada no âmbito das investigações de negócios, tais como investigações de mercado e estudos de caso. Dentre as técnicas de amostragem não probabilísticas existentes, a opção será pelo uso da não probabilística intencional heterogênea, em que se utiliza do julgamento para se proceder à escolha dos indivíduos a serem entrevistados. Relativamente aos procedimentos para coleta de dados primários, serão adotadas a observação participante e a entrevista semiestruturada. Quanto à análise, será realizada a análise qualitativa indutiva tendo-se em vista que os procedimentos de coleta adotados originam dados expressos em palavras.

Modelos são representações simplificadas da realidade; podem ser construídos como artefatos intelectuais (Mayr, 2007). Como simplificação da realidade, um modelo pode ser usado como ferramenta na tentativa de descrever, explicar e simular o funcionamento de um sistema. Seja por redução da complexidade, procurando a sua essência, seja por alteração na escala para mais ou para menos, o modelo desconsidera aspectos que não são relevantes para o objetivo da análise (Blanchard \& Fabrycky, 1981).

O modelo de análise proposto para a investigação em curso combina elementos presentes em quatro modelos teóricos clássicos das áreas de estratégia empresarial e do comportamento organizacional: os modelos de Porter (1998, 2008) para a competitividade e para o cluster respectivamente; o modelo de Hosfstede (1980) para a análise organizacional com base na cultura organizacional; e o modelo de Shein (1984, 1985) para estudo da cultura organizacional.

O modelo da competitividade desenvolvido por Porter (2008) leva em conta que a disputa entre empresas no mercado não ocorre apenas entre as que fazem parte de um mesmo segmento da indústria, mas vai além, para abranger também os clientes, os fornecedores, os entrantes potenciais e os produtos substitutos. A baixa concorrência, a possibilidade de se contrapor à pressão de clientes e fornecedores, as dificuldades para que novas empresas ingressem no mercado e a falta de substitutos viáveis podem favorecer as empresas concorrentes de um segmento industrial. Por outro lado, a rentabilidade das empresas pode ser corroída pelo acirramento da concorrência, pelo poder de negociação de clientes e de fornecedores, pela facilidade para que novas empresas entrem no mercado e pelas vantagens oferecidas por produtos substitutos. Assim, para o autor, a rivalidade que existe em meio a essas cinco forças é que irá definir a estrutura da indústria e modelar a natureza das interações competitivas no setor.

O modelo de cluster também desenvolvido por Porter (1998) leva em conta que os clusters abrangem não apenas as empresas concorrentes, mas frequentemente, os 
fornecedores especializados, alguns canais de distribuição, agências governamentais, instituições de ensino e pesquisa e associações comerciais; considera que eles afetam a competitividade de três formas: aumentando a produtividade das empresas da região, direcionando e dando ritmo à inovação e estimulando a formação de novos negócios que fortalecem o próprio cluster.

O modelo para análise organizacional desenvolvido por Hofstede (1980, 2010) considera que seis dimensões de cultura organizacional diferenciariam os agrupamentos humanos: 1) distância do poder - relaciona-se ao grau em que as pessoas menos poderosas de uma sociedade aceitam que o poder é distribuído desigualmente; 2) individualismo versus coletivismo - retrata o quanto as sociedades são mais coletivas que individuais; 3) evitação à incerteza - distingue a sociedade em relação à percepção da incerteza como uma ameaça por parte dos seus indivíduos; 4) masculinidade versus feminilidade - analisa a propensão de uma sociedade a ter mais características atribuídas ao gênero masculino ou ao gênero feminino; 5) orientação de longo prazo versus orientação normativa de curto prazo - analisa a propensão de uma sociedade de manter ou não ligação com o passado ao lidar com os desafios do presente e do futuro; e, 6) pragmática versus normativa - analisa a tendência de uma sociedade de permitir gratificar, de forma relativamente livre os interesses humanos básicos, ou de suprimir a sua satisfação.

O modelo de cultura organizacional de Shein (1984) leva em conta os elementos integrantes da cultura organizacional e como essa funciona; propõe que a análise seja feita em três níveis: 1) ao nível dos artefatos visíveis; 2) ao nível dos valores que governam o comportamento dos indivíduos; e 3) ao nível dos pressupostos básicos. Ao nível dos artefatos visíveis é possível descrever como um grupo constrói o seu ambiente e quais os padrões de comportamento que diferecenciam os seus membros. Ao nível dos valores que são manifestados ou defendidos pelos indivíduos é possível identificar a expressão da razão, ou o porquê dos indivíduos se comportarem de determinado modo. Ao nível dos pressupostos básicos é possível determinar como os membros de um grupo percebem, pensam e sentem.

O modelo de análise proposto para esta investigação leva em conta os elementos que integram os quatro modelos anteriormente citados e inclui o fator cultura local como elemento focal à análise. O processo de coleta e análise de dados no estudo de cluster, à luz da cultura local, prevê o seguinte:

- Caracterização da cultura predominante entre os indivíduos da localidade na qual o cluster está inserido;

- Utilização do modelo de Porter (2008) para competitividade, para situar a indústria na qual o cluster se insere e verificar como ela se comporta em relação às forças competitivas que o mesmo sugere;

- Utilização do modelo de Porter (1998) para clusters, com vistas a identificar os diferentes elementos que constituem o cluster a ser analisado;

- Utilização do modelo de Hofstede (1980, 2010) para análise organizacional com base na cultura organizacional, de modo a identificar os aspectos que caracterizam a cultura organizacional no cluster a ser analisado; 
- Utilização do modelo de Schein (1984) para análise da cultura organizacional de modo a coletar dados e decifrar o paradigma cultural do cluster a ser analisado.

\section{Aplicação}

Segundo o SEBRAE (2013) no sul do Brasil, no estado de Santa Catarina, na região da Grande Florianópolis encontra-se um cluster formado por empresas de micro e pequeno porte ligadas à maricultura, que desenvolvem a atividade produtiva da malacocultura, ou cultivo de moluscos marinhos bivalves como ostras mexilhões e vieiras. A atividade produtiva desenvolvida nesse cluster existe a partir de aspectos ligados à origem açoriana e à pesca artesanal, e apresenta como resultado a geração de emprego e renda na região, a preservação da cultura local e o cuidado com o meio ambiente. Dentre os municípios que o integram, destaca-se o de Florianópolis, que isoladamente responde por cerca de $80 \%$ da produção brasileira da malacocultura.

Apesar da produção expressiva, do forte apoio institucional que recebe e das condições ambientais favoráveis, o cluster da malacocultura da Grande Florianópolis apresenta problemas. Em seu estudo, Minuzzi (2011) apresenta os principais pontos fracos do cluster na percepção dos seus membros de governança endógena, como a grande rivalidade que existe entre os produtores; a grande diversidade de interesses entre os membros; a falta de entendimento do conceito de cluster; a ausência de espírito de união, cooperação e associativismo entre os produtores; a dificuldade de comunicação entre os agentes; e a falta de método de trabalho para a condução de ações, entre outros.

Dentre os pontos fracos, ou entraves ao desenvolvimento do cluster da malacocultura da região da Grande Florianópolis apresentados por Minuzzi (2011), percebe-se que a maior parte está relacionada ao comportamento dos indivíduos, ou seja, à sua maneira de ser e agir, que, por sua vez, está condicionada pela sua cultura. Assim, por exemplo, a falta de cooperação, de união e de espírito associativo entre os produtores poderia ser entendida como uma manifestação de individualismo, quem sabe um traço da herança açoriana que colonizou a região, quem sabe uma característica comum aos produtores, desenvolvida ao longo de suas vidas como pescadores, já que muitos são originários da pesca artesanal, atividade extrativa e, muitas vezes, de caráter individual. Estas especificidades culturais locais podem afetar a competitividade das empresas no cluster, pela influência na cultura das organizações, ou pelo choque com a cultura empresarial, dificultando a gestão de negócios da produção.

A relação entre a questão geográfica e de localização e o sucesso das empresas foi estudada por Porter (1998). Ele observou que, em pleno processo de globalização, que reduz a importância relativa da localização, pela maior facilidade de trocas entre fornecedores e clientes de diferentes partes do mundo, ocorrem aglomerações de empresas de um mesmo setor em determinadas regiões, na forma de clusters industriais. Estas empresas tanto cooperam como competem entre si e, de acordo com o seu modelo de análise, além de condições geográficas comuns, compartilham fornecedores e clientes e o apoio de agências governamentais e de instituições de pesquisa e desenvolvimento. Com base no modelo de Porter, pode-se identificar que os principais fatores competitivos do cluster da malacocultura de Florianópolis são as 
águas de baía, favoráveis ao cultivo de ostras e mexilhões, e a proximidade de instituições como a Universidade Federal de Santa Catarina e a Empresa de Pesquisas Agropecuárias e Extensão Rural de Santa Catarina, que, respectivamente, fornecem as sementes, que são o principal insumo de produção, e proporcionam assistência técnica. As empresas organizadas em clusters tendem a ter uma melhor visão do mercado em conjunto do que quando isoladas e, podem não somente observar melhor as oportunidades de inovação, como têm a capacidade e a flexibilidade para agir mais rapidamente. As interconexões e a complementaridade no cluster resultam num todo maior que a soma das partes.

Em relação à competitividade das organizações, pode-se reconhecer que a competição não ocorre apenas entre empresas concorrentes em um mesmo segmento da indústria. Porter (2008) observou que ela abrange também os clientes, os fornecedores, os entrantes potenciais e os produtos substitutos. Os clientes disputam por preços mais baixos e produtos de melhor qualidade; os fornecedores por preços mais altos e por limitações na qualidade e nos serviços; os entrantes, concorrentes potenciais, pela possibilidade de conquistar uma fatia do mercado, e; os produtos substitutos, soluções alternativas para a mesma função, pela oportunidade de conquistar um novo mercado. A rivalidade que existe em meio a estas cinco forças é que irá definir a estrutura da indústria e modelar a natureza das interações competitivas no setor. Ao abordar, com base neste modelo de análise, as forças competitivas que atuam sobre as empresas do cluster da malacocultura da Grande Florianópolis, o estudo permite identificar fraquezas e oportunidades no posicionamento das empresas diante do mercado e compreender aspectos que envolvem o ambiente externo às organizações.

Hofstede (1980) define cultura organizacional como uma programação coletiva da mente humana, que diferencia os membros de um grupo ou categoria social, dos membros de outros grupos ou categorias sociais. Em sua definição o autor busca mostrar que o comportamento individual é previsível, já que para ele cada indivíduo recebe um programa mental, ou seja, uma cultura, que conduz o seu comportamento nas diversas situações da vida. Ao utilizar de forma combinada às seis abordagens que o autor propõe em seu modelo de análise organizacional com base na cultura organizacional, o estudo permite compreender aspectos da organização do cluster da malacocultura da Grande Florianópolis condicionados pela cultura organizacional.

Ao investigar a cultura organizacional, deve-se inicialmente admitir que ela encontrase na mente de todos os membros da organização e não apenas na de seus dirigentes. Para Schein (1984, 1985), a cultura organizacional é um padrão de pressupostos básicos que um dado grupo inventou, descobriu ou desenvolveu ao aprender a lidar com os problemas de adaptação externa e de integração interna. Esse padrão teria funcionado bem o suficiente para ser considerado válido e ensinado a novos membros como a maneira correta de perceber, pensar e sentir em relação aos problemas de adaptação. Porém não basta entender a cultura como um conjunto de significados compartilhados, mas é preciso saber como a cultura surge, como se torna o que é, e como mudá-la caso a sobrevivência da organização disso dependa. Para a tarefa de decifrar a cultura organizacional, o autor propõe que a análise seja feita em diferentes níveis: ao nível dos artefatos visíveis; ao nível dos valores que governam o comportamento dos indivíduos; e, ao nível dos pressupostos básicos. Como os valores são difíceis de ser observados diretamente, é necessário inferi-los por meio de 
entrevistas aos membros-chaves da organização, ou pela análise do conteúdo de artefatos, tais como documentos e cartas. Assim, ao utilizar o modelo de análise da cultura organizacional levando em cota as quatro abordagens possíveis segundo o autor, o estudo permite identificar aspectos da cultura organizacional e decifrar o paradigma cultural do cluster da malacocultura da região da Grande Florianópolis.

A amostra da investigação é composta por representantes dos diversos atores e segmentos que integram o cluster da malacocultura da Grande Florianópolis: empresários que exploram a atividade produtiva; empregados de empresas que exploram a atividade produtiva; produtores autônomos; instituições de apoio à atividade produtiva da malacocultura como UFSC e EPAGRI; Serviço Brasileiro de Apoio às Micro e Pequenas Empresas (SEBRAE); Secretaria Municipal da Pesca de Florianópolis e Instituto de Geração de Oportunidade de Florianópolis (IGEOF); fornecedores de insumos; empresas do seguimento de meios de hospedagem; clientes tais como proprietários de restaurantes da região e comerciantes do Mercado Público Municipal de Florianópolis.

A justificativa para adoção do cluster da malacocultura da região Grande Florianópolis como objeto de estudo da investigação que está em curso e de que trata este artigo consiste no fato do mesmo abranger cinco dos treze municípios que integram região da Grande Florianópolis, nos quais há predomínio de colonização açoriana. Além disso, nesses mesmos municípios onde a malacocultura se desenvolve, a origem dessa atividade produtiva foi determinada pelo mesmo fator em todos eles, isto é, o seu surgimento foi uma alternativa à pesca artesanal que se encontrava em fase de decadência na região.

A investigação tem como objetivo identificar as manifestações da cultura ou expressões da cultura, presentes na maneira de ser, pensar e sentir dos indivíduos que tenham implicações na cultura organizacional das empresas que integram o cluster e que se reflitam no seu desempenho. É preciso analisar aspectos relativos à percepção dos indivíduos quanto ao seu papel e sua influência nos resultados da atividade produtiva (individual e coletivo daquilo que fazem e da análise do que é feito) e os sentimentos em relação a isso.

O processo de coleta de dados será por meio de observação participante e de entrevistas semiestruturadas a serem realizadas com representantes dos diversos agentes que participam do cluster, como empresários, produtores autônomos, empregados na atividade da malacocultua, representantes de instituições participantes do cluster, representantes de empresas correlatas e, também, fornecedores e clientes do cluster.

Os dados serão ordenados de acordo com os modelos de análise de Porter (1998, 2008), Hofstede (1980) e Schein (1984, 1985). A análise dos dados irá destacar, nas diversas manifestações, os aspectos ligados às especificidades da cultura local, em contraste com os conteúdos ligados à cultura empresarial, para identificar as convergências e discrepâncias, os padrões e as singularidades nestas manifestações. 


\section{Conclusões}

A adoção de Saunders et al. (2012) como base para a abordagem metodológica da investigação de que se trata neste trabalho, possibilita estruturar a coleta e análise de dados. Evidenciar as escolhas metodológicas é uma forma de reflexão que possibilita aumentar significativamente a consistência do modelo da investigação.

A apresentação sistematizada da racionalização para as decisões assumidas no processo de definição metodológica contribui para justificar uma possível opção por coletar e analisar dados de forma qualitativa, bem como para reforçar a validade científica de tal escolha.

Para a área da Engenharia, um trabalho desta natureza pode servir para chamar a atenção de que até mesmo trabalhos que abordam temas subjetivos ou aspectos em que medições não são possíveis, ou são desnecessárias, têm como se submeter ao rigor metodológico para que se alcance consistência em seus resultados.

\section{Agradecimentos}

Os autores gostariam de agradecer o apoio do centro de investigação CGIT da Universidade do Minho. Este trabalho é apoiado por fundos FEDER através do Programa COMPETE e por fundos nacionais da FCT no âmbito do projeto PestE/EME/UIo252/2012.

\section{Referências}

Baird, K., Harrison G. \& Reeve, R. (2007). The culture of Australian organizations and its relations with strategy. International Journal of Business Studies, vol. 15, pp. 15-41. Jun.

Barney, J. B. (1986). Strategic factor markets: expectations, luck and business strategy. Management Science, vol. 32, pp. 1231-1241, Oct.

Blanchard, B. \& Fabrycky, W. (1981). Systems engineering and analysis. New Jersey, PrenticeHall.

Dickson, M. W., Adityia, R. N. \& Chhokar, J. S. (2000). Definition and interpretation in cross-cultural organizational culture research in Ashkanasy, N. M., Wilderom, C. P. M. \& Peterson, M. F. (eds), Handbook of organizational culture and climate. Thousand Oaks, Sage Publications.

Fairbanks, M. \& Lindsay, S. (2000). Arando o mar: fortalecendo as fontes ocultas de crescimento em países em desenvolvimento. Rio de Janeiro: Qualitymark.

Feldmann, P. R. (2010). A influência da cultura na gestão das empresas latinoamericanas. Estudos Avançados USP, vol. 24, pp. 321-334, 2010.

Hofstede, G. (1980) Culture's consequences: international differences in work-related values. Beverly Hills: Sage.

Hofstede, G. \& Minkov, M. (2010). Cultures and Organizations: Software of the Mind. 3rd Edition, USA McGraw-Hill. 
House, R. J., Quigley, N. R. \& deLuque, M.S. (2010). Insight from Project GLOBE: Extending global advertising research through a contemporany framework. International Journal of Advertising, 29 (1), 111-139.

Lenartowicz, T. \& Roth, K.(2001). Does subculture within a country matter? A crosscultural study of motivational domains and business performance in Brazil. Journal of International Business Studies, Washington, 32(2), 305-325.

Mariotto, F. L. (1991). O conceito de competitividade da empresa: uma análise crítica. Revista de Administração de Empresas. São Paulo, 31 (2) 37-52.

Martins, E. C. \& Terblanche, F. (2003). Building organisational culture that stimulates creativity and innovation. European Journal of Innovation Management, vol. 6, pp. $64-74$.

Mayr, L. R. (2007). Modelo da participação do cliente na produção por encomenda. Tese (Doutorado). Universidade Federal de Santa Catarina, Programa de PósGraduação em Engenharia de Produção. Florianópolis, Brasil.

McSweeney, B. (2009). Dynamic diversity: variety and variation within countries. Organization Studies. Berlin, 30 (9), 933-957.

Minuzzi, J. (2011). Desenvolvimento de metodologia para identificar competências da governança endógena de arranjos produtivos locais. Tese (doutorado). Programa de Pós-Graduação em Engenharia de Produção, Universidade Federal de Santa Catarina.

Porter, M. E.(1998). Clusters and the new economics of competition. Harvard Business Review, vol. 76, pp. 77-90, Nov.1998.

Porter, M .E. (2008). The five competitive forces that shape strategy. Harvard Business Review, vol 76, pp. 79-93, Jan.

Saunders, M., Lewis, P. \& Thornhill, A. (2012). Research Methods for Business Students. 6th ed., Harlow: Pearson Education Limited.

Schein, E. H. (1984). Coming to a new awareness of organizational culture. Sloan Management Review, vol. 25, pp. 3-16, Jan.

Schein, E. H. (1985). Organizational culture and leadership. San Francisco: JosseyBass.

SEBRAE (2013). Malacocultura. [Online]. Available: <http://www.sebraesc.com.br/leis/default.asp?vcdtexto $=5865 \&^{\wedge \wedge}>$ Access: 02/10/2013.

Yin, R.K. (2009). Case study research: design and methods. 4th ed. USA: Sage. 